\title{
Initial cerebrospinal fluid-restricted oligoclonal bands associate with anti- $N$-methyl-D-aspartate receptor encephalitis severity: a pilot study
}

\author{
Sang Bin Hong ${ }^{1}$, Yong-Won Shin ${ }^{2}$, Jangsup Moon ${ }^{3}$, Woo-Jin Lee ${ }^{1}$, Kon Chu ${ }^{1}$, Sang Kun Lee ${ }^{1}$ \\ ${ }^{1}$ Department of Neurology, Seoul National University Hospital, Seoul, Korea \\ ${ }^{2}$ Department of Neurosurgery, Center for Hospital Medicine, Seoul National University Hospital, Seoul, Korea \\ ${ }^{3}$ Rare Disease Center, Seoul National University Hospital, Seoul, Korea
}

\section{Purpose}

Intrathecal antibody production is thought to underly the pathogenesis and symptomatology of $N$-methyl-D-aspartate receptor encephalitis (NMDARE). In the present study, the clinical correlation of cerebrospinal fluid (CSF) restricted oligoclonal bands (OCBs), as a measure of intrathecal antibody synthesis, was examined in confirmed NMDARE cases.

\section{Methods}

The present study included patients with a confirmed diagnosis of NMDARE who underwent initial CSF evaluation and were followed up for a minimum of 12 months. Disease severity was assessed at baseline and 1, 3, 6, 9, and 12 months. Data regarding duration of hospitalization and intensive care unit (ICU) stay, the presence of uncontrolled seizures, and antiepileptic drug requirement were obtained for each patient.

\section{Results}

Among the 14 confirmed NMDARE patients, seven had CSF-OCBs. The presence of CSF-OCBs was associated with a more severe disease at baseline $(p=0.004)$, worse final outcome $(p=0.005)$, and longer hospitalization (median, 19 vs. 173 days; $p<0.001)$ and ICU stay (median, 0 vs. 29 days; $p=0.006)$. CSF-OCB positivity was closely associated with treatment refractoriness within 4 weeks $(p=0.029)$.

\section{Conclusion}

The presence of CSF-OCBs at the onset of disease in NMDARE patients was associated with initial treatment refractoriness and a more severe disease course leading to longer hospitalization, ICU admission, intractable seizures, and a poorer outcome. The results indicate that CSF-OCBs may be useful for prognostication. Furthermore, severe disease in NMDARE may be accompanied by oligoclonal expansion antibody-producing B cells.

Keywords: Autoimmune limbic encephalitis, Oligoclonal bands, Anti-N-methyl-D-aspartate receptor encephalitis

\section{Introduction}

With the increasing availability of antibody assays and clinical awareness of autoimmune encephalitides as a diagnosis of encephalitis, a growing literature exists on the diverse range of clinical manifestations of autoimmune encephalitides. One of the most commonly identified autoimmune encephalitis is anti- $N$-methyl-D-aspartate receptor encephalitis (NMDARE). NMDARE symptoms can range from seemingly benign to life-threatening [1-4]. Reliable biomarkers predictive of disease course at symptom onset would facilitate the administration of

Received: November 2, 2020 Accepted: October 9, 2020

Correspondence: Kon Chu

Department of Neurology, Seoul National University Hospital, 101 Daehak-ro, Jongno-gu, Seoul 03080, Korea

E-mail: stemcell.snu@gmail.com

ORCID: https://orcid.org/0000-0001-5863-0302

Copyright (C) 2021 by The Korean Encephalitis and Neuroinflammation Society

This is an open access article distributed under the terms of the Creative Commons Attribution Non-Commercial License (http://creativecommons.org/licenses/by-nc/4.0/) which permits unrestricted non-commercial use, distribution, and reproduction in any medium, provided the original work is properly cited. 
timely immunotherapy and adequate medical management of complications.

Previous prognostication scores, such as the anti-NMDA Receptor Encephalitis One-Year Functional Status (NEOS) score [5], have utilized various indices of disease severity to predict functional outcome. The prognostic potential of various baseline variables, symptoms, and clinical assessments in NMDARE have been evaluated in other studies $[6,7]$. However, despite many efforts to determine factors associated with prognosis in NMDARE, a more reliable biomarker with fewer practical limitations is currently needed [8].

Prior research indicates the pathology in NMDARE is directly mediated by the intrathecal presence of anti-NMDA receptor (NMDAR) antibodies, leading to the possibility that patient cerebrospinal fluid (CSF) may provide relevant information for predicting disease severity. However, commonly tested CSF parameters such as leukocyte count or protein level inconsistently correlate with prognosis $[9,10]$. Other CSF tests such as anti-NMDAR antibody titers [11] have a more limited applicability in the clinical setting.

CSF-restricted oligoclonal bands (CSF-OCBs) are present in various immune-mediated neurologic disorders, such as multiple sclerosis (MS), and central nervous system (CNS) infections. In MS in particular, diagnostic and prognostic use of CSF-OCBs has long been established [12], with more than two or three CSF-OCBs signifying dissemination in time and portending a severe disease course with more frequent relapses. In the NMDARE setting, CSF-OCB, as a sensitive and standard measure of intrathecal antibody synthesis, may also reflect disease activity and prognosis associated with expansion of clonal intrathecal antibody production.

In the present study, the clinical correlation of the presence of CSF-OCBs was examined and its potential value as a prognostic biomarker in NMDARE evaluated.

\section{Methods}

\section{Subjects}

The present study included patients with a definite diagnosis of NMDARE according to the criteria previously published [13], and subjects who underwent initial CSF evaluation, including CSF-OCB testing at our hospital. The patients were followed up for a minimum of 12 months. Informed written consent was obtained from each patient or patient guardian, and the study was approved by the Institutional Review Board at Seoul National University Hospital (No. 1204-078-406).

\section{Diagnosis of anti-NMDARE}

NMDARE was diagnosed based on clinical features, brain magnetic resonance imaging (MRI), CSF analysis, electroencephalography, and presence of anti-NMDAR antibodies in the CSF or serum. A commercial cell-based immunochemistry method and indirect fluorescence assay using human embryonic kidney 293 cells (Euroimmun AG, Lübeck, Germany) was used to screen for antibodies to NMDAR as previously described in the literature [14].

\section{OCB testing}

The presence of CSF-OCBs was tested at initial presentation with CSF and paired serum sampling using gel electrophoresis and isoelectric focusing with immunofixation. Positive results included an oligoclonal response of more than two bands in the CSF with the paired serum showing a normal polyclonal response, and a CSF oligoclonal pattern of different isoelectric points compared with the serum. Negative results included a polyclonal response in both CSF and sera, a mirror type response with similar OCBs in CSF and sera, or a monoclonal response typical for paraproteins.

\section{Clinical outcomes}

Modified Rankin Scale (mRS) and clinical assessment scale in autoimmune encephalitis (CASE), as previously detailed [15], were used to assess each patient at baseline, and 1, 3, 6, 9, and 12 months, and last follow-up time points. Information on the length of intensive care unit (ICU) stay and hospitalization was also collected. To estimate the severity of seizures, information on antiepileptic drug (AED) prescription and seizure control was obtained based on retrospective review of medical records.

For each patient, the NEOS score [5], a 5-point maximum score comprising ICU admission, treatment delay of $>4$ weeks, lack of clinical response to treatment within 4 weeks, MRI abnormality, and elevated CSF leukocyte count $>20$ cells, was calculated based on retrospective review of medical records. The relationship between NEOS score and OCB components was examined. The performance of the NEOS score and OCB predicting 12-month outcomes (mRS 0-1: no impairment, mRS 2-5: impairment) was compared.

\section{Statistical analyses}

Statistical analyses were performed using R 3.6.1 (R Foundation for Statistical Computing, Vienna, Austria). Wilcoxon rank-sum test, Fisher exact test, and Spearman correlation test were used as appropriate. A p-value of $<0.05$ was considered statistically significant. 
Table 1 Baseline characteristics of the study population

\begin{tabular}{|c|c|c|c|}
\hline Characteristic & CSF-OCB-negative $(n=7)$ & CSF-OCB-positive $(n=7)$ & p-value \\
\hline Age (yr) & $24(19-45)$ & $27(22-44)$ & 0.95 \\
\hline Female sex & $5(71.4)$ & $5(71.4)$ & $>0.999$ \\
\hline CSF sampling after IVlg treatment $^{a}$ & $4(57.1)$ & $5(71.4)$ & $>0.999$ \\
\hline IgG index & $0.51(0.29-3.69)$ & $0.83(0.22-2.20)$ & 0.95 \\
\hline CSF leukocytes (cells/ $\mu \mathrm{L})$ & $36(2-221)$ & $26(0-150)$ & 0.84 \\
\hline Symptom duration at CSF sampling (day) & $16(6-49)$ & $35(12-65)$ & 0.08 \\
\hline Follow-up duration (mo) & $16(10-28)$ & $21(19-77)$ & 0.17 \\
\hline Treatment delay > $4 \mathrm{wk}$ & $2(28.6)$ & $3(42.9)$ & $>0.999$ \\
\hline Teratoma & $2(28.6)$ & $3(42.9)$ & $>0.999$ \\
\hline
\end{tabular}

Values are presented as median (range) or number (\%).

CSF, cerebrospinal fluid; CSF-OCB, CSF-restricted oligoclonal band; IV, intravenous; Ig, immunoglobulin.

${ }^{a}$ Number of patients. ${ }^{b}$ Calculated using (CSF IgG/serum IgG)/(CSF albumin/serum albumin).

\section{Results}

\section{Clinical characteristics}

Fourteen confirmed NMDARE patients received initial CSFOCB testing. Differences in age, sex, and symptom duration at CSF-OCB testing were not observed (Table 1). Nine patients (four CSF-OCB-negative patients and five CSF-OCB-positive patients) underwent CSF testing after intravenous immunoglobulins had been started.

Among the patients, seven were positive for CSF-OCBs (Figure 1) and seven were negative. Significant differences in the initial CSF findings between CSF-OCB-negative and CSF-OCB-positive groups including CSF leukocyte count and immunoglobulin $\mathrm{G}$ (IgG) index were not observed (Table 1). IgG index (baseline: mRS [ $p=0.61]$, CASE [ $p=0.54$ ]; 12 months: $\operatorname{mRS}[p=0.37$ ], CASE $[\mathrm{p}=0.77]$ ) or the CSF leukocyte count (baseline: $\mathrm{mRS}$ [p = 0.95], CASE [ $p=0.92] ; 12$ months: $\mathrm{mRS}$ [ $\mathrm{p}=0.80$ ], CASE [ $\mathrm{p}=$ 0.89]) correlated with initial and follow-up clinical severity.

The follow-up duration after testing between CSF-OCB-negative and CSF-OCB-positive groups did not differ significantly, and differences in the proportion of patients receiving delayed immunotherapy were not observed (Table 1). All patients received multiple courses of first-line, second-line, and other immunotherapies, and teratoma removal in case of teratoma discovery, as previously detailed [16].

\section{CSF-OCB positivity at disease onset is associated} with marked differences in the initial severity and overall disease course

The mRS and CASE scores were significantly higher in CSF-OCB-positive patients than in CSF-OCB-negative patients at baseline, and 1, 3, 6, 9, and 12 months (Figure 2). Notably, an initial worsening of median CASE scores was observed in CSF-OCB-posi-
Figure 1 Patterns of multiple CSF, CSF-OCBs in anti-N-methylD-aspartate receptor antibody encephalitis patients

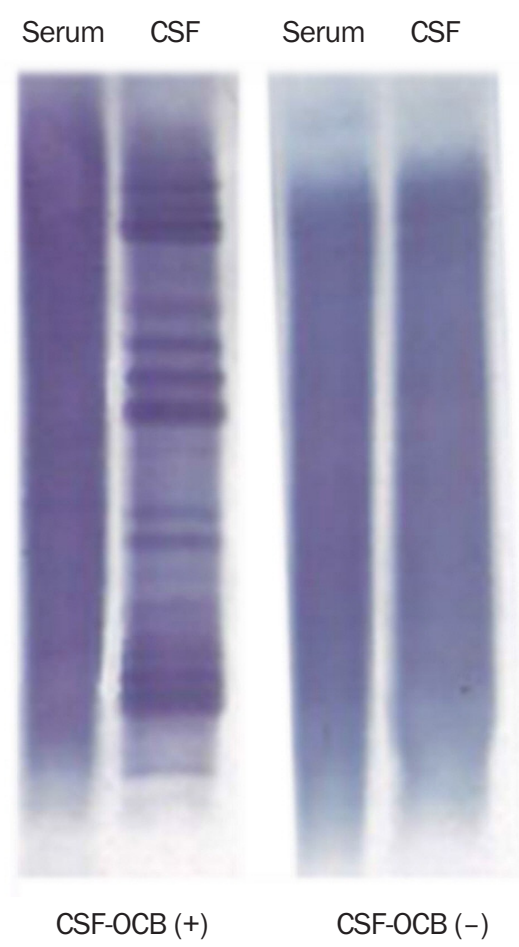

Gel electrophoresis, isoelectric focusing with immunofixation results of paired sera and CSF samples.

CSF, cerebrospinal fluid; CSF-OCB, CSF-restricted oligoclonal band.

tive patients at 1 month after presentation, contrary to the rapid reduction in CASE scores in CSF-OCB-negative patients. In CSF-OCB-negative patients, the disease course was favorable and no patient had remaining sequela impairing daily life. 

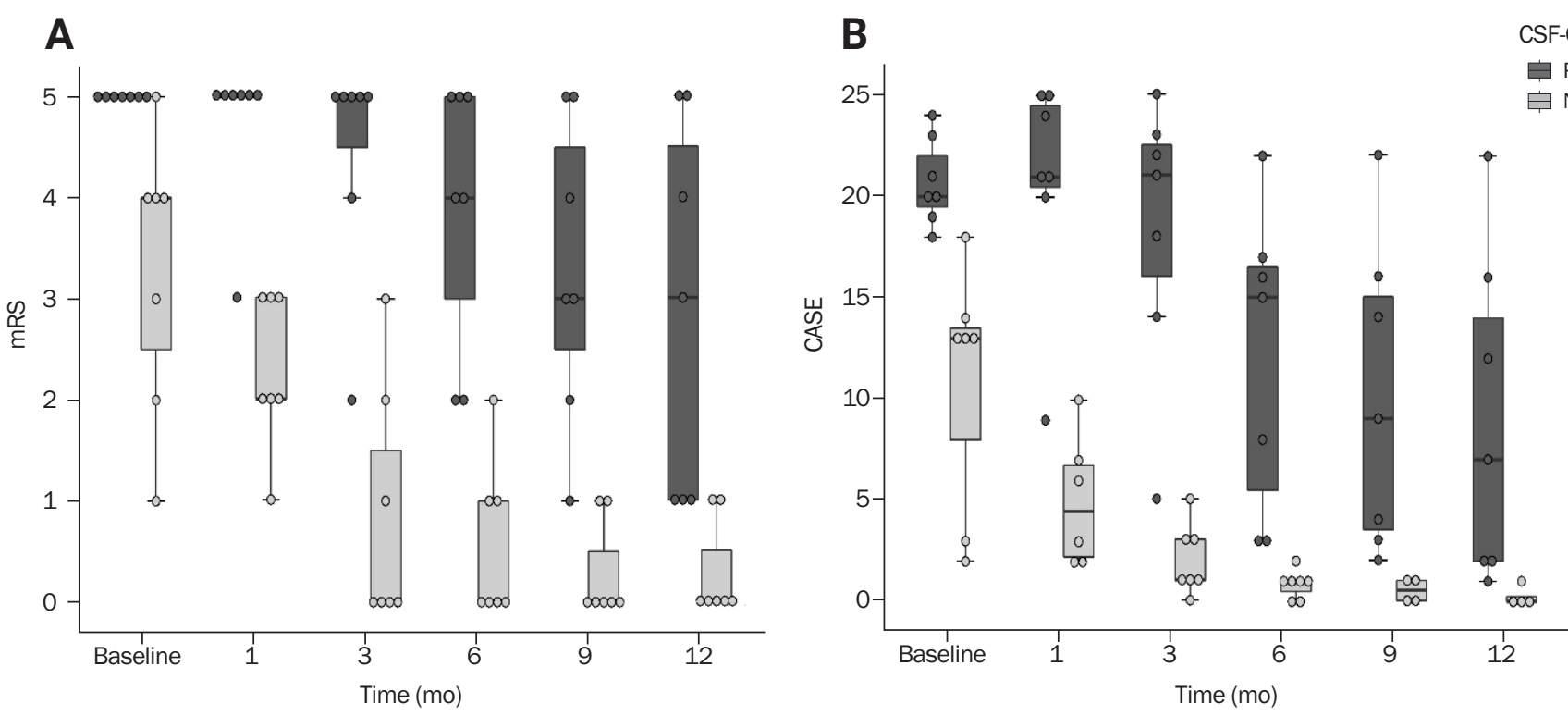

(A) Modified Rankin Scale (mRS). (B) Clinical assessment scale in autoimmune encephalitis (CASE).

CSF-OCB, cerebrospinal fluid-restricted oligoclonal band.

Table 2 Comparison of outcome variables between CSF-OCB-negative and CSF-OCB-positive groups

\begin{tabular}{|c|c|c|c|}
\hline Variable & CSF-OCB-negative $(n=7)$ & CSF-OCB-positive $(n=7)$ & p-value \\
\hline Total hospital stay (day) & $19(12-34)$ & $173(44-421)$ & $<0.001$ \\
\hline Total ICU stay (day) & $0(0-2)$ & $29(0-54)$ & 0.006 \\
\hline NEOS score & $2(0-3)$ & $3(2-4)$ & 0.008 \\
\hline Lack of treatment response in first $4 \mathrm{wk}$ & $1(14.3)$ & $6(85.7)$ & 0.029 \\
\hline ICU admission & $1(14.3)$ & $6(85.7)$ & 0.029 \\
\hline MRI abnormality & $3(42.9)$ & $4(57.1)$ & $>0.999$ \\
\hline CSF leukocytes > 20/ $\mu \mathrm{L}$ & $3(42.9)$ & $4(57.1)$ & $>0.999$ \\
\hline Modified Rankin Scale & & & 0.004 \\
\hline At baseline & $4(1-5)$ & $5(5-5)$ & \\
\hline At $12 \mathrm{mo}$ & $0(0-1)$ & $3(1-5)$ & 0.005 \\
\hline \multicolumn{4}{|l|}{ CASE } \\
\hline At baseline & $13(2-18)$ & $20(18-24)$ & 0.002 \\
\hline At $12 \mathrm{mo}$ & $0(0-1)$ & $7(1-22)$ & 0.013 \\
\hline Seizure severity ${ }^{\mathrm{a}}$ & $1(0-2)$ & $3(2-3)$ & 0.002 \\
\hline Maximum number of AEDs & $3(0-5)$ & $6(3-7)$ & 0.028 \\
\hline
\end{tabular}

Values are presented as median (range) or number (\%).

CSF, cerebrospinal fluid; CSF-OCB, CSF-restricted oligoclonal band; ICU, intensive care unit; NEOS, anti-NMDA Receptor Encephalitis One-Year Functional Status; MRI, magnetic resonance imaging; CASE, clinical assessment scale in autoimmune encephalitis; AED, antiepileptic drug.

aSeizure severity: 0 , no seizure; 1 , controlled seizures; 2 , uncontrolled seizures; 3 , status epilepticus.

CSF-OCB positivity predicts prolonged hospital stay and ICU admission

CSF-OCB positivity was associated with a longer duration of hospitalization ( $\mathrm{p}<0.001)$ and ICU stay $(\mathrm{p}=0.006$, Table 2$)$. Only one CSF-OCB-negative patient was admitted to the ICU and five of seven CSF-OCB-positive patients stayed in the ICU longer than 2 weeks. 


\section{CSF-OCB positivity indicates an increased risk of} seizures

CSF-OCB-positive patients had more severe seizures and required administration of multiple AEDs (Table 2). No CSFOCB-negative patient experienced status epilepticus; however, five CSF-OCB-positive patients experienced convulsive or nonconvulsive status epilepticus with four requiring a trial of six or more concomitant administrations of AEDs. After 1 year of follow-up, four of seven CSF-OCB-positive patients remained dependent on multiple AEDs and only one CSF-OCB-negative patient was on a single AED.

\section{Presence of CSF-OCBs is predictive of important NEOS score components}

CSF-OCB-positive patients were more likely to be admitted to the ICU ( $p=0.029)$ and have a lack of response to treatment in the first 4 weeks ( $p=0.029$ ) than CSF-OCB-negative patients. Significant relationship was not observed between the presence of CSF-OCBs and MRI abnormality or CSF leukocyte count. Both NEOS score and CSF-OCB positivity predicted outcome at 12 months equally well (sensitivity, 100\%; specificity, $64 \%$; area under the receiver operating characteristic curve, 0.82 ).

\section{Discussion}

In our NMDARE cohort, half of the patients were CSF-OCB-positive, somewhat comparable with previously reported prevalence ranging from $50 \%$ to $67 \%[17,18]$. When the clinical correlation of CSF-OCB positivity was examined, the results showed a close association with early treatment refractoriness and ICU admission, greater clinical severity, and an initially deteriorating clinical course leading to prolonged hospital stays, uncontrolled seizures, and poorer outcome.

Other candidate prognostic markers have also been proposed. Anti-NMDAR antibody titers in the CSF were shown partially associated with clinical outcome [11] as well as other measurable factors such as cell-free CSF mitochondrial DNA [19], NLRP3 inflammasome levels [20], Th17 cells [21], and cytokines [22]; however, routinely tested CSF parameters were shown to not correlate with disease trajectory $[9,10]$, as was the case in our cohort. CSF-OCB testing is advantageous because the test can be performed while a diagnosis of NMDARE is only suspected, and is widely available with an established method useful in many neurologic conditions.

Among the items of the NEOS score, treatment refractoriness in the first 4 weeks and ICU admission were the two strongest predictors of outcome [5]. Both factors closely paralleled CSF-OCB positivity, and CSF-OCB positivity alone performed equally well as the NEOS score in predicting 1-year outcomes in our cohort.

The pathomechanistic implications of our results may be notable for two reasons. First, although in previous literature the focus was on a single antibody targeting the GluN1 subunit of the NMDAR as being pathogenic [23-25] and responsible for most symptoms [26-29] in NMDARE, the concomitant presence of oligoclonal antibodies appear to have clinical relevance. In MS, CSF-OCBs are thought to be produced by clonally expanded B cells within the intrathecal space [30] as a result of somatic hypermutation of $\mathrm{B}$ cells upon continued antigen presentation [31]. Therefore, CSF-OCBs in NMDARE could be a result of continued and prolonged exposure to antigens in the CNS triggering persistent immune response. This also may be the reason early removal of teratoma improves prognosis in NMDARE [32] and indicates multiple related antigenic targets that intensify disease pathology may exist in a portion of NMDARE patients. Another possibility is that CSF-OCBs reflect direct damage to the CNS and release various types of neural and glial antigens due to severe disease activity, and are not the cause.

Second, intrathecal humoral immune response appears closely associated with clinical severity in NMDARE. Among CSFOCB-negative patients, the majority had a normal polyclonal pattern in both serum and CSF (five of seven; two had single bands in the CSF), and among CSF-OCB-positive patients, six of seven showed a normal polyclonal response in the serum. Thus, a distinct immune profile within the CNS was correlated with severe symptomatology, indicating intrathecal humoral immune response is primarily responsible for determining the clinical trajectory of NMDARE.

Lastly, a few issues should be considered when interpreting these results. Initial symptom severity in our cohort delineated patients with severe disease course from patients with milder disease course. Because clinical evaluations were made at similar time points as the lumbar puncture, any additional prognostic value beyond the initial clinical evaluation may be disputable. However, the CSF-OCBs have a practical advantage because they are a single diagnostic and prognostic biomarker, requiring no additional expert interpretation.

Further limitations of this study include the small sample size and the tertiary care setting with limited generalizability. However, the drastically contrasting clinical course of CSF-OCB-negative and CSF-OCB-positive patients that was clearly demonstrated in our small cohort, warrants further studies in which the relationship between CSF-OCB and clinical severity is investi- 
gated.

In conclusion, the present study results indicate initial CSF-OCB testing in NMDARE may aid in identifying patients with unfavorable disease course and clearly stratifying patients into a severe and a benign group. Larger prospectively designed studies will help determine the clinical utility of initial OCB testing in NMDARE.

\section{Conflicts of Interest}

Jangsup Moon, Kon Chu, Sang Kun Lee have been editorial board of encephalitis since October 2020. They were not involved in the review process of this original article. No other potential conflict of interest relevant to this article was reported.

\section{Author Contributions}

Conceptualization: SB Hong, YW Shin, K Chu, SK Lee; Data curation, Formal analysis, Project administration, Investigation: SB Hong; Methodology: SB Hong, YW Shin; Resources: K Chu, SK Lee; Supervision: YW Shin, J Moon, WJ Lee, K Chu, SK Lee; Writing-original draft: SB Hong; Writing-review and editing: SB Hong, YW Shin, J Moon.

\section{Acknowledgments}

This work was supported by the National Research Foundation of Korea(NRF) grant funded by the Korea government (MSIT) (No. 2020R1C1C1014982).

\section{References}

1. Dalmau J, Lancaster E, Martinez-Hernandez E, Rosenfeld MR, Balice-Gordon R. Clinical experience and laboratory investigations in patients with anti-NMDAR encephalitis. Lancet Neurol 2011;10:63-74.

2. de Montmollin E, Demeret S, Brulé N, et al. Anti-N-methyl-d-aspartate receptor encephalitis in adult patients requiring intensive care. Am J Respir Crit Care Med 2017;195:491-499.

3. Lim JA, Lee ST, Jung KH, et al. Anti-N-methyl-d-aspartate receptor encephalitis in Korea: clinical features, treatment, and outcome. J Clin Neurol 2014;10:157-161.

4. Wandinger KP, Saschenbrecker S, Stoecker W, Dalmau J. Anti-NMDA-receptor encephalitis: a severe, multistage, treatable disorder presenting with psychosis. J Neuroimmunol 2011;231:86-91.

5. Balu R, McCracken L, Lancaster E, Graus F, Dalmau J, Titulaer MJ. A score that predicts 1-year functional status in patients with anti-NMDA receptor encephalitis. Neurology 2019;92:e244-e252.
6. Irani SR, Bera K, Waters P, et al. N-methyl-D-aspartate antibody encephalitis: temporal progression of clinical and paraclinical observations in a predominantly non-paraneoplastic disorder of both sexes. Brain 2010;133:1655-1667.

7. Titulaer MJ, McCracken L, Gabilondo I, et al. Treatment and prognostic factors for long-term outcome in patients with anti-NMDA receptor encephalitis: an observational cohort study. Lancet Neurol 2013;12:157-165.

8. Dalmau J, Armangué T, Planagumà J, et al. An update on anti-NMDA receptor encephalitis for neurologists and psychiatrists: mechanisms and models. Lancet Neurol 2019;18:1045-1057.

9. Schubert J, Brämer D, Huttner HB, et al. Management and prognostic markers in patients with autoimmune encephalitis requiring ICU treatment. Neurol Neuroimmunol Neuroinflamm 2018;6:e514.

10. Wang R, Guan HZ, Ren HT, Wang W, Hong Z, Zhou D. CSF findings in patients with anti-N-methyl-D-aspartate receptor-encephalitis. Seizure 2015;29:137-142.

11. Gresa-Arribas N, Titulaer MJ, Torrents A, et al. Antibody titres at diagnosis and during follow-up of anti-NMDA receptor encephalitis: a retrospective study. Lancet Neurol 2014;13:167-177.

12. Arrambide G, Tintore M, Espejo C, et al. The value of oligoclonal bands in the multiple sclerosis diagnostic criteria. Brain 2018; 141:1075-1084.

13. Graus F, Titulaer MJ, Balu R, et al. A clinical approach to diagnosis of autoimmune encephalitis. Lancet Neurol 2016;15:391-404.

14. Shin YW, Lee ST, Kim TJ, Jun JS, Chu K. Bortezomib treatment for severe refractory anti-NMDA receptor encephalitis. Ann Clin Trans Neurol 2018;5:598-605.

15. Lim JA, Lee ST, Moon J, et al. Development of the clinical assessment scale in autoimmune encephalitis. Ann Neurol 2019;85:352-358.

16. Shin YW, Lee ST, Park KI, et al. Treatment strategies for autoimmune encephalitis. Ther Adv Neurol Disord 2017;11:1756285617722347.

17. Blinder T, Lewerenz J. Cerebrospinal fluid findings in patients with autoimmune encephalitis-a systematic analysis. Front Neurol 2019;10:804.

18. Dalmau J, Gleichman AJ, Hughes EG, et al. Anti-NMDA-receptor encephalitis: case series and analysis of the effects of antibodies. Lancet Neurol 2008;7:1091-1098.

19. Peng Y, Zheng D, Zhang X, et al. Cell-free mitochondrial DNA in the CSF: a potential prognostic biomarker of anti-NMDAR encephalitis. Front Immunol 2019;10:103.

20. Peng Y, Liu B, Pei S, et al. Higher CSF levels of NLRP3 inflammasome is associated with poor prognosis of anti-N-methyl-D-Aspartate receptor encephalitis. Front Immunol 2019;10:905.

21. Zeng C, Chen L, Chen B, et al. Th17 cells were recruited and accumulated in the cerebrospinal fluid and correlated with the poor prognosis of anti-NMDAR encephalitis. Acta Biochim Biophys Sin (Shanghai) 
2018;50:1266-1273.

22. Liba Z, Kayserova J, Elisak M, et al. Anti-N-methyl-D-aspartate receptor encephalitis: the clinical course in light of the chemokine and cytokine levels in cerebrospinal fluid. J Neuroinflammation 2016;13:55.

23. Castillo-Gómez E, Oliveira B, Tapken D, et al. All naturally occurring autoantibodies against the NMDA receptor subunit NR1 have pathogenic potential irrespective of epitope and immunoglobulin class. Mol Psychiatry 2017;22:1776-1784.

24. Hughes EG, Peng X, Gleichman AJ, et al. Cellular and synaptic mechanisms of anti-NMDA receptor encephalitis. J Neurosci 2010;30:58665875 .

25. Moscato EH, Peng X, Jain A, Parsons TD, Dalmau J, Balice-Gordon RJ. Acute mechanisms underlying antibody effects in anti-N-methyl-D-aspartate receptor encephalitis. Ann Neurol 2014;76:108-119.

26. Jones BE, Tovar KR, Goehring A, et al. Autoimmune receptor encephalitis in mice induced by active immunization with conformationally stabilized holoreceptors. Sci Transl Med 2019;11:eaaw0044.

27. Kreye J, Wenke NK, Chayka M, et al. Human cerebrospinal fluid monoclonal N-methyl-D-aspartate receptor autoantibodies are sufficient for encephalitis pathogenesis. Brain 2016;139:2641-2652.

28. Ly LT, Kreye J, Jurek B, et al. Affinities of human NMDA receptor autoantibodies: implications for disease mechanisms and clinical diagnostics. J Neurol 2018;265:2625-2632.

29. Wenke NK, Kreye J, Andrzejak E, et al. N-methyl-D-aspartate receptor dysfunction by unmutated human antibodies against the NR1 subunit. Ann Neurol 2019;85:771-776.

30. Obermeier B, Mentele R, Malotka J, et al. Matching of oligoclonal immunoglobulin transcriptomes and proteomes of cerebrospinal fluid in multiple sclerosis. Nat Med 2008;14:688-693.

31. Qin Y, Duquette P, Zhang Y, Talbot P, Poole R, Antel J. Clonal expansion and somatic hypermutation of $\mathrm{V}(\mathrm{H})$ genes of B cells from cerebrospinal fluid in multiple sclerosis. J Clin Invest 1998;102:1045-1050.

32. Seki M, Suzuki S, Iizuka T, et al. Neurological response to early removal of ovarian teratoma in anti-NMDAR encephalitis. J Neurol Neurosurg Psychiatry 2008;79:324-326. 